\title{
From Dark to Bright: First-Order Perturbation Theory with Analytical Mode Normalization for Plasmonic Nanoantenna Arrays Applied to Refractive Index Sensing
}

\author{
T. Weiss, ${ }^{*}$ M. Mesch, M. Schäferling, and H. Giessen \\ 4th Physics Institute and Research Center SCoPE, University of Stuttgart, Pfaffenwaldring 57, D-70550 Stuttgart, Germany \\ W. Langbein and E. A. Muljarov \\ Cardiff University, School of Physics and Astronomy, The Parade, CF24 3AA Cardiff, United Kingdom
}

(Received 21 August 2015; published 8 June 2016)

\begin{abstract}
We present a first-order perturbation theory to calculate the frequency shift and linewidth change of photonic resonances in one- and two-dimensional periodic structures under modifications of the surrounding refractive index. Our method is based on the resonant state expansion, for which we extend the analytical mode normalization to periodic structures. We apply this theory to calculate the sensitivity of bright dipolar and much darker quadrupolar plasmonic modes by determining the maximum shift and optimal sensing volume.
\end{abstract}

DOI: 10.1103/PhysRevLett.116.237401

Whenever we can relate concentration changes of relevant substances in a material to changes of the refractive index, resonantly enhanced refractive index sensing provides an all-optical technique to measure the concentration [1-8], as depicted in Fig. 1(a). In recent years, this has become one of the key applications of plasmonics and photonic crystals.

As the changes of the refractive index can be rather small, common numerical simulations are prone to failure. This is because extremely small changes in the refractive index environment of nanostructures will only lead to minute shifts and linewidth changes of the optical resonances that serve as the transducer for the refractive index change to be measured. Particularly, small perturbations require a high numerical accuracy, rendering a solely numerical analysis rather inefficient.

In contrast, a first-order perturbation theory is ideally suited for these cases [2,3,9-12]. Such a perturbation theory relies on calculating the resonances of the unperturbed system, including an appropriate normalization of the resonant field distributions. In time-independent perturbation theory for bound states in quantum mechanics [13], the normalization is carried out such that integrating the absolute square of the eigenfunctions over the whole space becomes unity. This approach, however, is not generally applicable in optics. The main difficulty is that optically active resonances exhibit radiative losses, resulting in modes that are diverging towards the far field [14] [see Fig. 1(c)]. Therefore, an integral over the whole space diverges. Significant work has been devoted to developing suited normalization procedures [15-26].

We present here a first-order perturbation theory using an analytical mode normalization. Our approach follows from the more general resonant state expansion $[18,22,23]$ and allows thorough analysis and simplified optimization of refractive index sensor designs. As an example we show results for a periodic nanoantenna array, which is directly compared with experiment [27]. The presented approach can be adapted to other geometries such as single nanoantennas.

In this Letter, we show the main steps to derive the perturbation equation for periodic structures at normal incidence with details given in the Supplemental Material [27]. Furthermore, we apply the theory to analyze dipolar (DP) and quadrupolar (QP) plasmonic modes for refractive index sensing by determining optimal sensing volumes and the role of mutual coupling between nanoantennas.
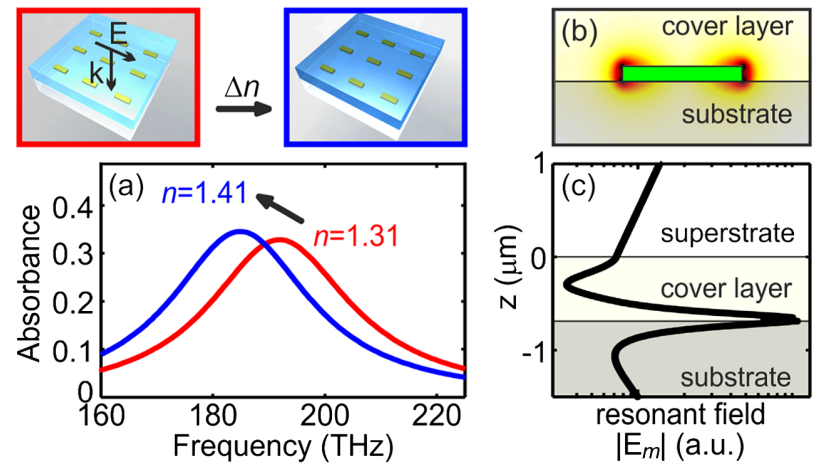

FIG. 1. (a) Principle of refractive index sensing with a periodic array of gold nanoantennas: a cover layer serves as the sensing volume, in which the refractive index $n$ changes with the concentration of an analyte. This results in a frequency shift of the plasmonic resonances in the nanoantennas, which can be detected optically; see the absorbance spectra calculated at normal incidence for $n=1.31$ (red) and $n=1.41$ (blue). (b) Cross section through the center of the antenna (one unit cell) with the magnitude $\left|\mathbf{E}_{m}\right|$ of the resonant electric field for $n=1.31$ as the background color. (c) $\left|\mathbf{E}_{m}\right|$ at the center of a unit cell. The fields grow exponentially in the top and bottom half-spaces. 
The perturbation theory precisely and efficiently predicts linewidth and resonance frequency changes due to small changes of the refractive index, without the need to repeatedly solve Maxwell's equations. The method works with any numerical technique that allows for calculating optical eigenmodes from Maxwell's equations. Our numerical calculations are based on the Fourier modal method [32,33] with adaptive coordinates [34-37].

In the frequency domain [Gaussian units, $\mu=1$, spatially dependent permittivity $\varepsilon(\mathbf{r})]$, the optical wave equation reads

$$
\mathcal{L}(\omega) \mathbf{E} \equiv-\boldsymbol{\nabla} \times \boldsymbol{\nabla} \times \mathbf{E}+k_{0}^{2} \varepsilon \mathbf{E}=-i k_{0} \frac{4 \pi}{c} \mathbf{j},
$$

where $\mathcal{L}$ is the corresponding differential operator, $k_{0}=\omega / c$, and $\mathbf{E}$ denotes the electric field at frequency $\omega$ due to the source $\mathbf{j}$. A time dependence $\exp (-i \omega t)$ is understood. For $\mathbf{j}=0$ and outgoing boundary conditions, Eq. (1) defines an eigenvalue equation for eigenfrequencies $\omega_{m}$ and resonant field distributions $\mathbf{E}_{m}$, which is $\mathcal{L}\left(\omega_{m}\right) \mathbf{E}_{m}=0$. In general, $\omega_{m}$ is a complex number with $\operatorname{Im}\left(\omega_{m}\right) \leq 0$. The real part specifies the resonance frequency, while $-2 \operatorname{Im}\left(\omega_{m}\right)$ defines the linewidth.

We can express the Green's dyadic of $\mathcal{L}$ in terms of its resonances [18,22,23]. For periodic systems obeying Lorentz reciprocity, one obtains at normal incidence

$$
\mathcal{G}\left(\mathbf{r}, \mathbf{r}^{\prime} ; \omega\right)=c^{2} \sum_{m} \frac{\mathbf{E}_{m}(\mathbf{r}) \otimes \mathbf{E}_{m}\left(\mathbf{r}^{\prime}\right)}{2 \omega_{m}\left(\omega-\omega_{m}\right)}+\Delta \mathcal{G}\left(\mathbf{r}, \mathbf{r}^{\prime} ; \omega\right) .
$$

Here, $\otimes$ denotes the vector direct product, $\Delta \mathcal{G}\left(\mathbf{r}, \mathbf{r}^{\prime} ; \omega\right)$ is defined as the contribution of the discontinuities due to Rayleigh-Wood anomalies [23], and the sum is carried out over all resonances on the complex frequency plane. Equation (2) requires a proper normalization of $\mathbf{E}_{m}$, which is given by Eq. (3) below. It has been derived by adapting the normalization procedure of Refs. [22,38] to periodic systems.

Equation (2) allows developing a linear perturbation theory based on the resonant state expansion, where we consider a modified differential operator $\mathcal{L}_{\Delta \varepsilon}$, in which $\varepsilon$ is replaced by $\varepsilon+\Delta \varepsilon$. Then, we rewrite the wave equation such that the deviation from $\mathcal{L}$ may be considered as the source term. Thus, for $\mathcal{L}_{\Delta \varepsilon}$, resonant field distributions $\mathbf{E}_{\mu}$ with eigenvalues $\omega_{\mu}$ satisfy $\mathcal{L}\left(\omega_{\mu}\right) \mathbf{E}_{\mu}=-\omega_{\mu}^{2} \Delta \varepsilon\left(\omega_{\mu}\right) \mathbf{E}_{\mu} / c^{2}$. By convoluting the Green's dyadic of $\mathcal{L}$ with the right-hand side of this equation, we can derive an implicit equation for $\mathbf{E}_{\mu}$. If $\Delta \varepsilon$ is sufficiently small [27], we may assume that $\mathbf{E}_{\mu} \approx \mathbf{E}_{m}$, which allows us to calculate the modified eigenvalues $\omega_{\mu}=\omega_{m}+\Delta \omega_{m}$ in the first-order approximation.

In the case that $\Delta \varepsilon$ is restricted to a sensing volume $\mathcal{T}$, in which $\varepsilon=n^{2}$ and $\Delta \varepsilon$ are spatially constant, we can derive the resonance shift for refractive index changes $\Delta n$ in the limit $\Delta n \rightarrow 0$.
Perturbation theory: $\frac{\partial \omega_{m}}{\partial n}=-\omega_{m} n \int_{\mathcal{T}} d V \mathbf{E}_{m}^{2}(\mathbf{r})$.

$$
\text { Normalization: } 1=I_{m}^{\mathcal{V}}+S_{m}^{\text {top }}+S_{m}^{\text {bot }} \text {. }
$$

Here, $I_{m}^{\mathcal{V}}$ is a volume integral over volume $\mathcal{V}$, which spans over one unit cell and includes the interfaces to the top and bottom half-spaces:

$$
I_{m}^{\mathcal{V}}=\left.\int_{\mathcal{V}} d V \mathbf{E}_{m} \cdot \frac{\partial\left(\omega^{2} \varepsilon\right)}{\partial\left(\omega^{2}\right)}\right|_{\omega_{m}} \mathbf{E}_{m}
$$

Importantly, the normalization contains surface contributions, which can be written at normal incidence as

$$
S_{m}^{\nu}=\left.\frac{i}{2} \frac{\partial\left(\omega^{2} \varepsilon\right)}{\partial\left(\omega^{2}\right)}\right|_{\omega_{m}} \sum_{\mathbf{G}, p} \frac{1}{\kappa_{\mathbf{G}}^{\nu}} a_{m,-\mathbf{G}}^{(p, \nu)} a_{m, \mathbf{G}}^{(p, \nu)},
$$

where $\nu$ indicates either top or bottom outer half-space. To arrive at Eq. (5), we have used a plane wave expansion in the outer half-spaces, which is $\mathbf{E}_{m}^{(\nu)}(\mathbf{r})=$ $\sum_{\mathbf{G}, p} a_{m, \mathbf{G}}^{(p, \nu)} \mathbf{E}_{\mathbf{G}}^{(p, \nu)} e^{i \mathbf{G} \cdot \mathbf{r}+i \kappa_{\mathbf{G}}^{\nu} \Delta z}$, where $\Delta z=0$ on $\partial \mathcal{V}$ and $\Delta z>0$ outside $\mathcal{V} ; p$ labels linear polarization states obeying $S_{u} \mathbf{E}_{\mathbf{G}}^{(p)} \cdot \mathbf{E}_{-\mathbf{G}}^{(q)}=\delta_{p, q}\left(S_{u}\right.$ is the area of one unit cell), with $\mathbf{G}$ being reciprocal lattice vectors with components $G_{\alpha}=2 \pi g_{\alpha} / P_{\alpha}$ for periods $P_{\alpha}, g_{\alpha} \in \mathbb{Z}$, and in-plane directions $\alpha=1,2$. The $z$ component of the wave vectors is $\kappa_{\mathbf{G}}^{\nu}=\left(\varepsilon_{\nu} k_{0}^{2}-\mathbf{G}^{2}\right)^{1 / 2}$. Note that $\mathbf{E}_{m}^{2}(\mathbf{r})$ in Eq. (3) is proportional to the inverse of the averaged mode volume of a randomly oriented dipole at $\mathbf{r}$ [27].

Figure 2 shows a comparison of the results of the perturbation theory in Eq. (3) with the full numerical calculations for a periodic array of rectangular gold antennas. The antenna width, length, and thickness are

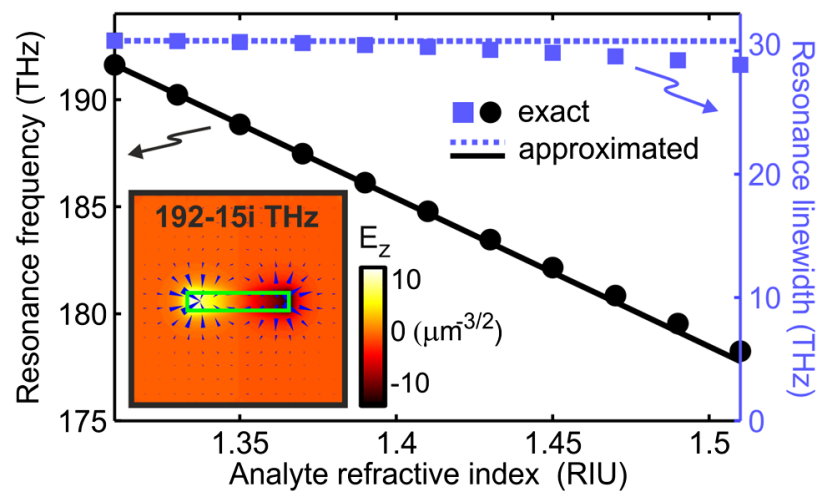

FIG. 2. Resonance frequency (black) and linewidth (blue) as a function of the refractive index in the sensing volume for a periodic system of gold rod antennas (spectra in Fig. 1). Solid and dotted lines: linear perturbation theory, see Eq. (3). Dots and squares: full numerical calculations. The inset shows the resonant field distribution in a unit cell. The background color depicts the $z$ component of the electric field $10 \mathrm{~nm}$ above the nanoantennas. RIU denotes refractive index units. 
60,330 , and $40 \mathrm{~nm}$, respectively, with a period of $700 \mathrm{~nm}$. The substrate is glass $(n=1.5)$. The antennas are covered by $650 \mathrm{~nm}$ of water $(n=1.31)$ as the sensing volume, and the superstrate is air. The gold has been modeled according to Ref. [39]. The absorbance spectra for normally incident plane waves with the electric field oriented along the long antenna axis can be seen in Fig. 1 for a varying refractive index in the sensing volume.

The numerical results (details in Ref. [27]) are shown as dots and squares in Fig. 2. Using the perturbation theory, we have to calculate the resonances only once in order to determine the linear change of both the resonance frequency and linewidth as a function of the refractive index in the sensing volume; see solid and dotted lines in Fig. 2. Our perturbation theory with an analytical normalization (calculated at $n=1.31$ ) agrees well with the full numerical results over the shown refractive index range. Specifically, it reproduces the linear slope around $n=1.31$ within the numerical accuracy of the full calculations.

Note that Eq. (3) can also be used to derive the mode normalization numerically from the resonance shift. In particular, calculating $\partial \omega_{m} / \partial n$ numerically and comparing it with Eq. (3), one can find the normalization of $\mathbf{E}_{m}$.

As a next step, we study plasmonic systems exhibiting DP and QP modes of similar resonance frequencies. The first structure is a $30 \mathrm{~nm}$ thick gold film with three slits per unit cell (substrate, superstrate, and cover layer as above, $P_{1,2}=800 \mathrm{~nm}$ ); see Ref. [5] and the schematic on top of Fig. 3(a). The slit pair providing the QP mode has a width of $90 \mathrm{~nm}$ and a length of $370 \mathrm{~nm}$; the slit for the DP resonance is $400 \mathrm{~nm}$ long and $80 \mathrm{~nm}$ wide. The gap between the QP and DP slits is $45 \mathrm{~nm}$.

The corresponding far-field spectra (incident polarization perpendicular to the DP slit, normal incidence) show a typical dipolar resonance if the DP slit is centered with regard to the QP slit pair (dashed lines). In this case, no interaction between the DP and QP modes is possible. The QP mode becomes visible as a Fano-type line shape on top
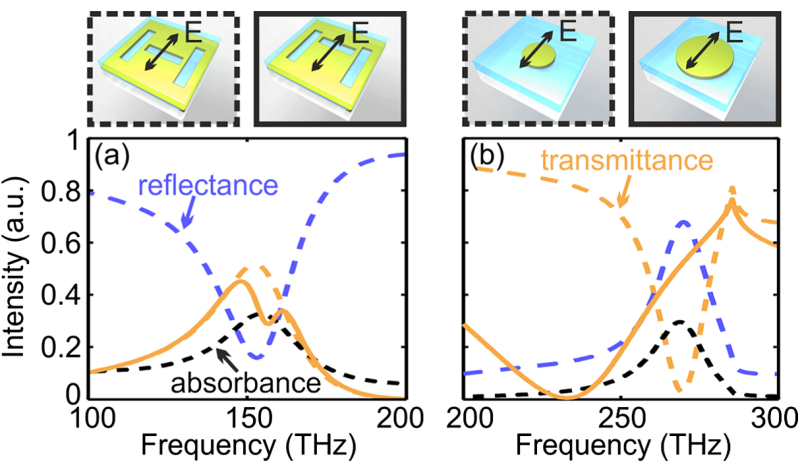

FIG. 3. Transmittance (orange), reflectance (blue), and absorbance (black) for a periodic array of (a) slit antennas in a gold film and (b) circular gold disks. The incident polarization and schematics are shown on top. The dashed (solid) lines denote the optical responses for the symmetric (asymmetric) slit configuration in (a) and smaller (larger) disks in (b). of the DP resonance $[9,40]$ if the DP slit is shifted by $30 \mathrm{~nm}$ (solid line). Note that our parameters are such that the DP and QP modes have similar resonance frequencies in the uncoupled configuration.

Figure 3(b) shows the far-field spectra for periodic arrays of gold disks with a thickness of $30 \mathrm{~nm}$ (other parameters as for the rod antennas): The solid lines denote disks of radius $210 \mathrm{~nm}$; the dashed lines show the results for a radius of $132 \mathrm{~nm}$. The radii have been chosen such that the QP resonance frequency of the larger disks agrees with the DP resonance frequency of the smaller disks.

For applications, an interesting quantity is the optimal sensing volume, i.e., how efficiently the refractive index changes can be detected in small volumes and at which sensing volume the resonance frequency shift saturates. This can be derived from Eq. (3) by limiting the test volume above the antennas to certain thicknesses. The corresponding resonance shifts are shown in Fig. 4. Furthermore, we define the sensing volume efficiency $\eta$ as

$$
\eta=\frac{1}{T} \int_{\mathcal{T}} d V \mathbf{E}_{m}^{2}
$$

where $T$ denotes the volume of the sensing region.

Analytical results for spherical particles [41] indicate that the near fields of higher-order modes decay more rapidly outside a plasmonic particle. Therefore, one would expect a better sensing volume efficiency of the QP mode as well as faster saturation of the resonance shift of the QP mode as compared to the DP mode. However, in Fig. 4(a), we see an almost identical behavior of both modes

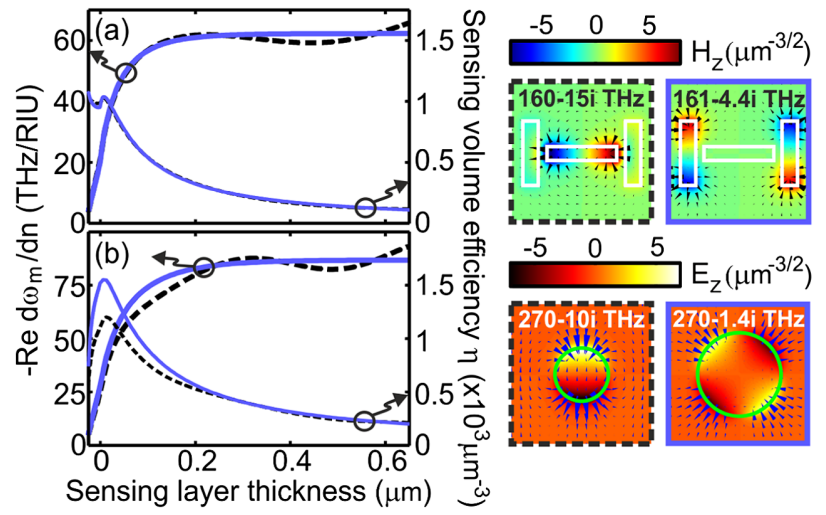

FIG. 4. Frequency shift per refractive index change (thick lines) as well as the sensing volume efficiency $\eta$ (thin lines) of the dipolar (black dashed) and quadrupolar (blue solid) modes calculated by Eq. (3) for (a) the gold film with a symmetric slit configuration and (b) gold disks of radius $132 \mathrm{~nm}$ (dipole) and $210 \mathrm{~nm}$ (quadrupole) as a function of the sensing volume thickness. The cover layer thickness is $650 \mathrm{~nm}$. Panels to the right show the resonant magnetic (top) and electric (bottom) field distributions $10 \mathrm{~nm}$ above the antennas for a sensing volume of $650 \mathrm{~nm}$ thickness. The background color denotes the corresponding $z$ components. Note that in our convention, a sensing layer thickness of $0 \mathrm{~nm}$ actually corresponds to changing the refractive index of the analyte in the slits or between the gold disks. 
TABLE I. Comparison of complex resonance frequency $\omega_{m}$, resonance shift $\partial \omega_{m} / \partial n$ derived as a difference quotient by full numerical calculations or Eq. (3) for sensing volumes of thickness $650 \mathrm{~nm}$, and the figure of merit for the different geometries.

\begin{tabular}{lcccr}
\hline \hline & $\omega_{m}$ & $\partial \omega_{m} / \partial n$ (THz/RIU) & FOM \\
& $(\mathrm{THz})$ & Numerical & Equation (3) & (a.u.) \\
\hline Rods & $191.6-15.4 i$ & $-70.8+0.3 i$ & $-69.2+0.1 i$ & 4.5 \\
Slits DP & $160.0-14.5 i$ & $-66.6+8.7 i$ & $-65.8+8.5 i$ & 4.6 \\
Slits QP & $161.4-4.4 i$ & $-61.8+0.1 i$ & $-62.2+1.1 i$ & 14.1 \\
Disks DP & $270.0-10.2 i$ & $-95.0-8.1 i$ & $-93.3-8.1 i$ & 9.3 \\
Disks QP & $270.3-1.4 i$ & $-88.2-1.6 i$ & $-86.8-1.7 i$ & 62.6 \\
\hline \hline
\end{tabular}

(symmetric configuration with zero shift of the DP slit), with saturation around $150 \mathrm{~nm}$ thickness. The reason is that in this geometry, the QP mode originates from an antisymmetric alignment of the DP fields. Hence, the near fields have the same dependence as those of the DP mode in the single slit. Differences occur only for larger thicknesses, where we observe an oscillatory behavior for the DP mode. In contrast, the QP mode in the larger disks exhibits a better sensing volume efficiency and saturation at smaller sensing volume thicknesses when compared to the results of the DP mode in the smaller disks. Therefore, regarding small sensing volumes, the QP mode in the disks is superior compared to the DP mode.

An advantage of the QP mode in the slit system is its narrow linewidth (see Table I). Neglecting any influence of the experimental setup [3] as well as the excitation efficiency of the modes, we consider the following figure of merit (FOM):

$$
\mathrm{FOM}=\left|\frac{1}{\operatorname{Im}\left(\omega_{m}\right)} \frac{\partial \omega_{m}}{\partial n}\right| .
$$

A comparison of resonance frequency shifts and the FOM can be found in Table I, revealing a good agreement between perturbation theory and the full numerical calculations. As shown in Ref. [27], the FOM is proportional to the Purcell factor, so that large field enhancements and high quality factors are beneficial for the sensing performance. The largest shifts can be obtained by the disk system, with a FOM of 62.6 for the QP mode.

In Eq. (3), we see that the resonance shift is proportional to the refractive index in the test volume as well as to the resonance frequency. Therefore, increasing the resonance frequency, e.g., by changing the period, should be beneficial. In contrast, a larger resonance linewidth decreases the FOM. In order to separate linewidth effects from shifts of the resonance frequency, we show in Fig. 5 the period dependence of the resonance frequencies [Fig. 5(b)], their refractive index sensitivities [Figs. 5(c) and 5(d)], and the FOM [Fig. 5(e)] for a constant disk radius (dashed lines) and for period-dependent radii [see Fig. 5(a)] such that the resonance frequency is independent of the period (solid lines).

Figure 5(b) shows two main contributions to the mutual coupling of the disks with identical radius but varying
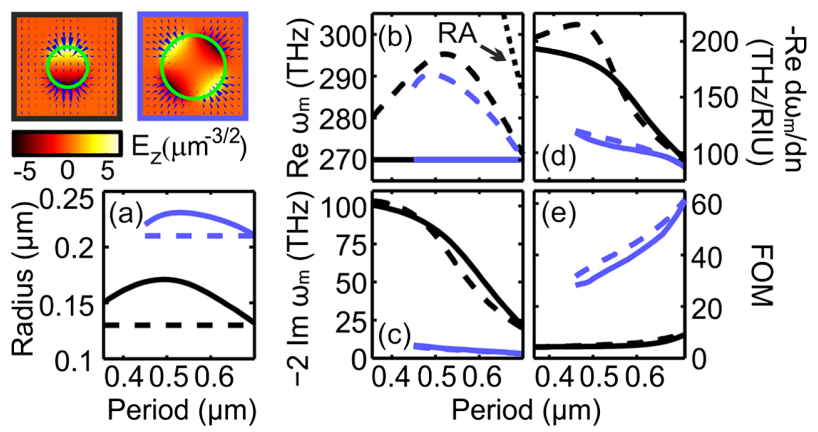

FIG. 5. Comparison of dipolar (black) and quadrupolar (blue) modes with similar resonance frequencies for different periods. (a) Radius for constant resonance frequency (solid lines); dashed lines indicate constant radius. (b) Resonance frequencies and (c) linewidths. (d) The frequency shift per refractive index change is stronger for the dipolar modes. (e) The figure of merit is higher for the quadrupolar mode and increases for larger periods. The dotted line in (b) denotes the Rayleigh anomaly (RA), which occurs due to the periodicity of the structures.

period. For small periods, the near-field coupling dominates, resulting in a decreased resonance frequency due to the energetically preferred charge configuration in the neighboring disks. For larger periods, the resonance frequency is decreased again due to an increased influence of the grating: at the Rayleigh anomalies [the dotted line in Fig. 5(b) denotes the first Rayleigh anomaly at the substrate interface], one or several plane waves in the exterior layers change their character normal to the top or bottom interface from decaying to propagating. Thus, at the Rayleigh anomaly, a plane wave is propagating exactly along the interface, mediating a stronger coupling of adjacent antennas and resulting in distinct spectral features [see Fig. 3(b) around $285 \mathrm{THz}$ ], which has been also utilized in sensing applications [42].

The linewidth [Fig. 5(c)] decreases monotonically as a lower density of disks results in a weaker coupling to the far field, i.e., smaller radiation damping. In Fig. 5(d), we depict the resonance frequency shift per refractive index change, calculated by Eq. (3). For the DP mode with varying radius (black solid line), we observe a monotonic behavior following the linewidth, while the influence of $\operatorname{Re}\left(\omega_{m}\right)$ can be seen for the DP mode with constant radius (black dashed line) by the presence of a maximum. For the QP modes (blue lines), the radius dependence is less pronounced. In the FOM [Fig. 5(e)], the linewidth effect dominates over the absolute shifts, rendering the QP modes preferable.

In summary, we present here an exact expression for the sensitivity of optical modes in planar one- and twodimensional periodic arrays at normal incidence. It uses a first-order approximation to the full resonant state expansion and the correct mode normalization for this geometry.

We illustrate the technique for systems with dipolar and quadrupolar modes, showing that quadrupolar modes constructed by an asymmetric superposition of dipoles are not 
superior regarding the sensing volume. The period dependence reveals that near-field coupling reduces the resonance shifts. Larger periods are beneficial due to a narrower linewidth. Finally, the mode normalization developed here will be relevant for exact expressions of the Purcell effect [43] in open systems [14,38]. Our approach can be also applied to describe resonance shifts for geometrical changes or modifications in dispersive materials $[44,45]$.

We acknowledge support from ERC Advanced Grant COMPLEXPLAS as well as DFG (SPP 1839), VW Stiftung, BMBF, MWK Baden-Württemberg (Zukunftsoffensive IV), and the Baden-Württemberg Stiftung (Spitzenforschung II). W. L. acknowledges support by his Leverhulme Royal Society Research Fellowship (Grant No. LT20085). E. A. M. and W. L. acknowledge support from EPSRC Impact Acceleration Account No. EP/ K503988/1 and the Sêr Cymru National Research Network.

*t.weiss@pi4.uni-stuttgart.de

[1] W. D. Wilson, Science 295, 2103 (2002).

[2] S. Arnold, M. Khoshsima, I. Teraoka, S. Holler, and F. Vollmer, Opt. Lett. 28, 272 (2003).

[3] A. Unger and M. Kreiter, J. Phys. Chem. C 113, 12243 (2009).

[4] K. M. Mayer and J.H. Hafner, Chem. Rev. 111, 3828 (2011).

[5] N. Liu, T. Weiss, M. Mesch, L. Langguth, U. Eigenthaler, M. Hirscher, C. Sönnichsen, and H. Giessen, Nano Lett. 10, 1103 (2010).

[6] P. Rapp, M. Mesch, H. Giessen, and C. Tarìn, Journal of Electrical and Computer Engineering 2011, 953064 (2011).

[7] A. E. Cetin, A. F. Coskun, B. C. Galarreta, M. Huang, D. Herman, A. Ozcan, and H. Altug, Light Sci. Appl. 3, e122 (2014).

[8] J. Prasad, I. Zins, R. Branscheid, J. Becker, A. H. R. Koch, G. Fytas, U. Kolb, and C. Sönnichsen, J. Phys. Chem. C 119, 5577 (2015).

[9] B. Gallinet, T. Siegfried, H. Sigg, P. Nordlander, and O. J. F. Martin, Nano Lett. 13, 497 (2013).

[10] W. Zhang and O. J. F. Martin, ACS Photonics 2, 144 (2015).

[11] J. Yang, H. Giessen, and P. Lalanne, Nano Lett. 15, 3439 (2015).

[12] F. Ruesink, H. M. Doeleman, R. Hendrikx, A. F. Koenderink, and E. Verhagen, Phys. Rev. Lett. 115, 203904 (2015).

[13] C. Cohen-Tannoudji, Quantum Mechanics (Hermann, Paris, 1991).

[14] A. F. Koenderink, Opt. Lett. 35, 4208 (2010).

[15] H. M. Lai, P. T. Leung, K. Young, P. W. Barber, and S. C. Hill, Phys. Rev. A 41, 5187 (1990).

[16] C. Sauvan, J. P. Hugonin, I. S. Maksymov, and P. Lalanne, Phys. Rev. Lett. 110, 237401 (2013).

[17] Q. Bai, M. Perrin, C. Sauvan, J. P. Hugonin, and P. Lalanne, Opt. Express 21, 27371 (2013).
[18] E. A. Muljarov, W. Langbein, and R. Zimmermann, Europhys. Lett. 92, 50010 (2010).

[19] P. T. Kristensen, C. V. Vlack, and S. Hughes, Opt. Lett. 37, 1649 (2012).

[20] M. B. Doost, W. Langbein, and E. A. Muljarov, Phys. Rev. A 85, 023835 (2012).

[21] M. B. Doost, W. Langbein, and E. A. Muljarov, Phys. Rev. A 87, 043827 (2013).

[22] M. B. Doost, W. Langbein, and E. A. Muljarov, Phys. Rev. A 90, 013834 (2014).

[23] L. J. Armitage, M. B. Doost, W. Langbein, and E. A. Muljarov, Phys. Rev. A 89, 053832 (2014).

[24] P. T. Kristensen, R.-C. Ge, and S. Hughes, Phys. Rev. A 92, 053810 (2015).

[25] E. A. Muljarov and W. Langbein, arXiv:1602.07278v1.

[26] E. A. Muljarov and W. Langbein, Phys. Rev. B 93, 075417 (2016).

[27] See Supplemental Material at http://link.aps.org/ supplemental/10.1103/PhysRevLett.116.237401, which includes Refs. [28-31], for details on the derivation of Eq. (3), the numerical calculation of the optical eigenmodes, as well as further numerical and experimental results for the rod antenna.

[28] R. G. Barrera, G. A. Estévez, and J. Giraldo, Eur. J. Phys. 6, 287 (1985).

[29] G. B. Arfken and H. J. Weber, Mathematical Methods for Physicists, 6th ed. (Elsevier, London, 2011).

[30] S. Kedenburg, M. Vieweg, T. Gissibl, and H. Giessen, Opt. Mater. Express 2, 1588 (2012).

[31] K. Moutzouris, M. Papamichael, S. Betsis, I. Stavrakas, G. Hloupis, and D. Triantis, Appl. Phys. B 116, 617 (2014).

[32] L. Li, J. Opt. A 5, 345 (2003).

[33] D. A. Bykov and L. L. Doskolovich, J. Lightwave Technol. 31, 793 (2013).

[34] T. Weiss, N. A. Gippius, S. G. Tikhodeev, G. Granet, and H. Giessen, J. Opt. A 11, 114019 (2009).

[35] T. Weiss, G. Granet, N. A. Gippius, S. G. Tikhodeev, and H. Giessen, Opt. Express 17, 8051 (2009).

[36] S. Essig and K. Busch, Opt. Express 18, 23258 (2010).

[37] T. Weiss, N. A. Gippius, S. G. Tikhodeev, G. Granet, and H. Giessen, J. Opt. Soc. Am. A 28, 238 (2011).

[38] E. A. Muljarov and W. Langbein, arXiv:1409.6877v3.

[39] P. G. Etchegoin, E. C. Le Ru, and M. Meyer, J. Chem. Phys. 125, 164705 (2006).

[40] B. Gallinet and O. J. F. Martin, Opt. Express 19, 22167 (2011).

[41] G. Mie, Ann. Phys. (Berlin) 330, 377 (1908).

[42] J. M. McMahon, J. Henzie, T. W. Odom, G. C. Schatz, and S. K. Gray, Opt. Express 15, 18119 (2007).

[43] E. M. Purcell, Phys. Rev. 69, 37 (1946).

[44] A. Tittl, X. Yin, H. Giessen, X.-D. Tian, Z.-Q. Tian, C. Kremers, D. N. Chigrin, and N. Liu, Nano Lett. 13, 1816 (2013).

[45] N. Strohfeldt, A. Tittl, M. Schäferling, F. Neubrech, U. Kreibig, R. Griessen, and H. Giessen, Nano Lett. 14, 1140 (2014). 